\title{
Frequency of repeat antinuclear antibody testing in Ontario: a population-based descriptive study
}

\author{
Shirley Lake MD MSc, Zhan Yao MD MS, Natasha Gakhal MD MSc, Amanda Steiman MD MSc, \\ Gillian Hawker MD MSc, Jessica Widdifield PhD
}

\section{Abstract}

Background: Repeat antinuclear antibody (ANA) testing may be unnecessary, potentially harmful and costly. Our aim was to assess the frequency and correlates of repeat ANA testing in Ontario.

Methods: We performed a retrospective descriptive study identifying ANA tests performed over 2008-2015 among adults within the Ontario Laboratories Information System. Our primary outcome was any ANA test performed within 1 year of a previous ANA test. Our secondary outcome was any repeat test after a previous positive result. Repeat testing overall (regardless of who performed the previous test) and repeat testing by the same provider who performed the previous test were determined separately. We assessed correlates of repeat testing (e.g., patient and physician characteristics) and of repeat testing after a positive result using separate logistic regression models by means of generalized estimating equations to account for clustering of repeat testing within patients and within physician practices.

Results: In total, 587357 ANA tests were performed in 437966 patients over the study period, of which 126322 (21.5\%) gave a positive result and $164913(28.1 \%)$ were repeat tests. Family physicians ordered 358422 tests (61.0\%), and rheumatologists ordered 65071 tests (11.1\%). Of the repeat tests, 82332 (49.9\%) were ordered within 12 months of the previous test. Among the 73961 repeat tests ordered by the same practitioner within 12 months, the previous test result was positive for 22657 (30.6\%). A higher proportion of rheumatologists than other physicians ordered repeat tests within 12 months (36.1\% v. 11.3\%). The most significant correlate of potentially redundant testing was testing among patients with suspected or confirmed connective tissue disease.

Interpretation: Over a quarter of ANA tests in Ontario were repeat tests; rheumatologists were most likely to order repeat testing. Our findings may be useful to inform quality-improvement initiatives related to the appropriateness of ANA testing.

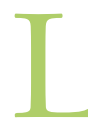

aboratory testing is the highest-volume medical procedure, ${ }^{1}$ and volumes are increasing annually. ${ }^{2,3}$ It has been estimated that $20 \%$ of tests are ordered unnecessarily. ${ }^{4,5}$ Misuse of laboratory tests is a major challenge affecting the sustainability of health care. ${ }^{6,7}$ Improving the appropriateness of rheumatology laboratory testing is a priority of Choosing Wisely campaigns. ${ }^{8,9}$

Concerns have been raised about the inappropriate use of antinuclear antibody (ANA) testing. Testing for ANA is indicated only if a patient's clinical history and physical examination show symptoms or signs suggestive of systemic lupus erythematosus, scleroderma, Sjögren syndrome, polymyositis or dermatomyositis. ${ }^{10,11}$ The test has high sensitivity, and, thus, a positive test result can contribute to a diagnosis of these conditions. ${ }^{12}$ However, it has low specificity, and ANA and can be seen in other conditions and in more than $20 \%$ of healthy people, ${ }^{13}$ which makes interpretation of test results challenging. ${ }^{12}$
Choosing Wisely Canada recommends that "ANA testing should not be used to screen subjects without specific symptoms or without a clinical evaluation that may lead to a diagnosis of systemic lupus or other connective tissue disease." International recommendations strongly advise that "ANA testing is primarily intended for diagnostic purposes, and not for monitoring disease progression" owing to its limited value in monitoring disease activity. ${ }^{14-17}$ Thus, it is not appropriate to repeat ANA following a positive test result..$^{7-9,16,18}$

\section{Competing interests: None declared.}

This article has been peer reviewed.

Correspondence to: Shirley Lake, Shirley.Lake@sunnybrook.ca CMAJ Open 2020. DOI:10.9778/cmajo.20190148 
Inappropriate testing may cause patients confusion and anxiety, and lead to overdiagnosis, overtreatment, and unnecessary consultations and costs. ${ }^{19-23}$ Moreover, given the rare incidence of systemic autoimmune rheumatic diseases ${ }^{24-26}$ and previous research suggesting that ANA tests are often ordered serially or in settings of low pretest probability, ${ }^{19,27,28}$ understanding patterns of ANA testing is useful to inform quality-improvement initiatives assessing the appropriateness of ANA testing.

Therefore, our aim in the present study was to assess the frequency and correlates of repeat ANA testing.

\section{Methods}

We performed a retrospective study over 2008-2015 using health administrative databases in Ontario.

\section{Sources of data}

We identified ANA tests (including dates, test results and ordering physician) using Logical Observation Identifiers Names and Codes from the Ontario Laboratories Information System, a nearly population-wide database of laboratory test results in Ontario. The Ontario Laboratories Information System captures both community and hospital laboratory tests. At the time of analysis, the period of laboratory data spanned from Jan. 1, 2007, to Sept. 30, 2015, and the provincial coverage increased from $41 \%$ in 2008 to $71 \%$ in 2009 , $86 \%$ in 2010 and $99 \%$ in 2014.

We linked patients with ANA tests performed between 2008 and 2015 to the Ontario Health Insurance Plan Claims Database to identify diagnoses (according to a modification of the 8 th revision of the International Classification of Diseases), and to the Canadian Institute for Health Information Discharge Abstract Database to identify hospital admissions. We identified patient demographic characteristics from the Registered Persons Database and ordering physician specialty by linking with the ICES Physician Database.

These data sets are linked by means of unique encoded patient and physician identifiers and are held securely and analyzed at ICES, a prescribed entity under section 45 of Ontario's Personal Health Information Protection Act.

\section{Participant eligibility}

Patients were excluded if they were less than 18 years of age, had missing patient or physician identifiers, lived out of province or died on the date of their first ANA test.

\section{Outcome measures}

Our primary outcome was any ANA test within 1 year of a previous ANA test. Our secondary outcome was any repeat ANA test after a previous positive test result.

\section{Covariates}

Patient-level covariates included age, sex, income quintile (as a proxy for socioeconomic status, based on patients' postal code and census neighbourhood income quintile), rural versus urban residence (defined with the Rurality Index for Ontario ${ }^{29}$ based on participants' postal code), year of testing, hospital admission in the 6 months before testing and Charlson Comorbidity Index score (computed with a 2-yr look-back period from the index test). As a proxy for testing or confirmation of connective tissue disease, we determined whether patients had at least 1 Ontario Health Insurance Plan diagnosis code for connective tissue disease (710 or 695) within a 1 -year period before or 6 months after the test date.

Physician-level covariates included specialty (rheumatologist, internist, family medicine or other), age, sex, whether they were international medical graduates, and whether they practised in an academic or community setting (determined by physician primary practice location based on postal codes linked to an academic hospital location).

\section{Statistical analysis}

We assessed the frequency of ANA testing at the health system level, patient level and provider level, and repeat testing for individual patients within 12 months of a previous ANA test. The frequency of total ANA tests performed, positive test results and repeat tests performed within 12 months of a previous test was determined overall and by ordering physician type. Repeat testing overall (regardless of who performed the previous test) and repeat testing by the same provider who ordered the previous test were determined separately.

For results at the level of ANA testing, we expressed percentages using the denominator for the total number of ANA tests. For patient-level results, the denominator included the total number of patients with at least 1 ANA test. We assessed intervals between testing in relation to preceding negative or positive test results. We compared characteristics between patients with 2 or more ANA tests and those with 1 test.

To assess correlates of repeat testing within 12 months of a previous test, and any repeat test in which the previous test result was positive, we fit 2 separate logistic regression models by means of generalized estimating equations to account for clustering of testing within patients and within physician practices in order to assess the unique contribution of the variables to each outcome. Models accounted for both physician-level and patientlevel characteristics (the aforementioned covariates). The results of the regression analysis were expressed as odds ratios (ORs) and their $95 \%$ confidence intervals (CIs), representing the population-averaged effects of covariates on each outcome of interest.

The primary analysis focused on all repeat testing (within $12 \mathrm{mo}$ ) irrespective of the provider who ordered the previous test. Finally, we performed a sensitivity analysis to assess correlates of repeat ANA testing confined to the same provider ordering the previous test.

\section{Ethics approval}

This study was authorized under section 45 of the Personal Health Information Protection Act, which does not require review by a research ethics board.

\section{Resullts}

We identified 456726 patients with ANA tests between 2008 and 2015, of whom 18760 were excluded because they were 
less than 18 years old $(n=18170)$ or lived out of province $(n=$ 562). The remaining 28 patients were excluded owing to invalid health card numbers and death occurring on the date of the index test.

\section{Testing-level results}

In total, 587357 ANA tests were performed over the study period, of which $164913(28.1 \%)$ were repeat tests during the study period and $82332(14.0 \%)$ were repeat tests within 12 months of a previous test (Table 1). Of the 587357 , $126322(21.5 \%)$ gave a positive result.

We identified 7084 physicians who ordered ANA testing, of whom 188 were rheumatologists and 4643 were family physicians (Table 1). Family physicians ordered 358422 tests (61.0\%), rheumatologists ordered 65071 tests $(11.1 \%)$, and internists ordered 26409 tests $(4.5 \%)$. The top specialties for the remaining physicians were gastroenterology (22 239 tests [3.8\%]), neurology (20 120 [3.4\%]), dermatology (16 331 [2.8\%]) and nephrology (14 484 [2.5\%]). Rheumatologists had the highest frequency of positive results, and more rheumatologists ordered repeat tests within 12 months of a previous test than other practitioners (Table 1).

In our sensitivity analysis, of the 164913 repeat tests, 94392 $(57.2 \%)$ were ordered by the same practitioner: $16373(17.3 \%)$ were ordered within 3 months of the previous test, and 73961 (78.4\%) were ordered within 12 months of the previous test (Figure 1). Among the repeat tests ordered within 12 months, the previous test result was positive for 22657 (30.6\%).

\section{Patient-level results}

The 587357 ANA tests were performed for 437966 patients (294 130 women [67.2\%]; mean age 52.4 [standard deviation 16.3] yr). Of the 437966 patients, 346282 (79.1\%) had 1 test, and 91684 (20.9\%) had more than 1 test: $63084(14.4 \%)$ had 2 tests, 17000 (3.9\%) had 3 tests, $5857(1.3 \%)$ had 4 tests, and $5743(1.3 \%)$ had 5 or more tests.

Of the 437966 patients, 74849 (17.1\%) had a positive result of their first test recorded in the data source. Of the
91684 patients who had more than 1 test, $61684(67.3 \%)$ had their first test result reported as negative; of the 61684,4641 $(7.5 \%)$ had a subsequent positive test result.

Compared to the patients with a single ANA test, there was a higher proportion of female patients (65.4\% v. $73.9 \%)$ and presence of suspected or confirmed diagnosis of connective tissue disease (3.9\% v. $11.4 \%$ ) among patients with multiple tests (Table 2).

Among the 63084 patients with 2 tests, we identified 43706 patients for whom a family physician ordered the initial test. Of the 43706,5461 (12.5\%) had their repeat test ordered by a rheumatologist, and 30168 (69.0\%) had their repeat test ordered by a family physician.

\section{Correlates of repeat testing}

Family physicians, internists and all other care practitioners were significantly less likely than rheumatologists to order repeat testing within 1 year or repeat testing after a positive test result (Table 3). When we confined our analyses to repeat testing ordered by the same physician, the ORs remained significant. Physician demographic characteristics did not appear to be significantly associated with repeat testing, with the exception that internationally trained medical graduates were less likely than Canadian medical graduates to order repeat tests (adjusted OR 0.81, 95\% CI 0.70-0.93) and to order repeat tests after a previous positive result (OR 0.75, 95\% CI 0.63-0.88).

Female patients, those with a higher socioeconomic status and those with more comorbidities were more likely to undergo repeat testing within 12 months. Patients with suspected or confirmed connective tissue disease were significantly more likely than those without such a diagnosis to undergo repeat testing within 12 months (OR 2.20, 95\% CI 2.01-2.41 for any physician; OR 3.08, 95\% CI 2.70-3.51 for the same physician). Patients with suspected or confirmed connective tissue disease were also 4-5 times (OR 4.18, 95\% CI 3.70-4.73 for any physician, OR 5.37, 95\% CI 4.69-6.14 for the same physician) more likely to undergo repeat testing after a previous positive result (Table 3).

Table 1: Frequency of total and repeat antinuclear antibody tests overall and by ordering physician type

\begin{tabular}{|c|c|c|c|c|c|}
\hline \multirow[b]{2}{*}{ Variable } & \multicolumn{5}{|c|}{ No. $(\%)$ of tests } \\
\hline & $\begin{array}{c}\text { Total } \\
n=7136\end{array}$ & $\begin{array}{c}\text { Family } \\
\text { physicians } \\
n=4643\end{array}$ & $\begin{array}{l}\text { Rheumatologists } \\
\qquad n=188\end{array}$ & $\begin{array}{l}\text { Internal medicine } \\
\quad n=313\end{array}$ & $\begin{array}{c}\text { Other } \\
n=1992\end{array}$ \\
\hline No. of tests (\% of total tests) & $587357(100.0)$ & $358422(61.0)$ & $65071(11.1)$ & $26409(4.5)$ & $137455(23.4)$ \\
\hline Positive result & $126322(21.5)$ & $64262(17.9)$ & $28393(43.6)$ & $5884(22.3)$ & $27783(20.2)$ \\
\hline $\begin{array}{l}\text { Repeat test within } 12 \text { mo of } \\
\text { previous test (regardless of who } \\
\text { ordered previous test) }\end{array}$ & $82332(14.0)$ & $32994(9.2)$ & 23507 (36.1) & $4707(17.8)$ & $21124(15.4)$ \\
\hline $\begin{array}{l}\text { Repeat test within } 12 \text { mo of } \\
\text { previous test ordered by same } \\
\text { practitioner type who ordered } \\
\text { previous test }^{*}\end{array}$ & $51411(8.8)$ & $25213(7.0)$ & $13093(20.1)$ & $1656(6.3)$ & $11071(8.0)$ \\
\hline
\end{tabular}




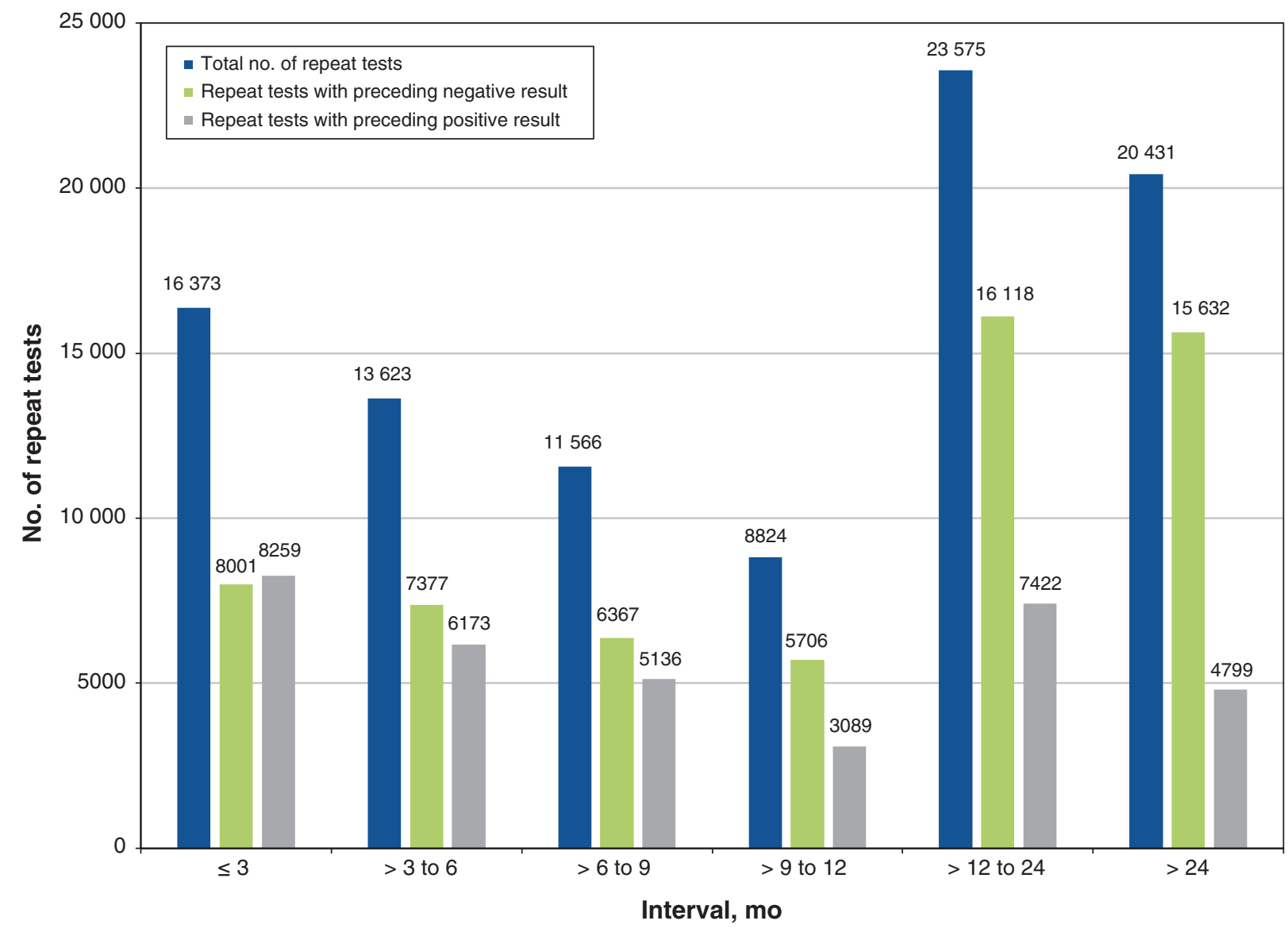

Figure 1: Number of repeat antinuclear antibody (ANA) tests ordered by the same practitioner, according to result and by time interval. A small proportion $(0.005 \%-0.8 \%)$ of tests had unknown results, and, therefore, the number of positive and negative results within each time interval may not add up to the cumulative total.

\begin{tabular}{|c|c|c|c|}
\hline \multirow[b]{2}{*}{ Characteristic } & \multicolumn{3}{|c|}{ No. $(\%)$ of patients* } \\
\hline & $\begin{array}{c}\text { Total } \\
n=437966\end{array}$ & $\begin{array}{c}1 \text { test } \\
n=346282\end{array}$ & $\begin{array}{c}\geq 2 \text { tests } \\
n=91684\end{array}$ \\
\hline Age, yr, mean \pm SD & $52.4 \pm 16.3$ & $51.9 \pm 16.5$ & $54.5 \pm 15.3$ \\
\hline Female sex & $294130(67.2)$ & $226363(65.4)$ & 67767 (73.9) \\
\hline Connective tissue disease & $24037(5.5)$ & $13610(3.9)$ & $10427(11.4)$ \\
\hline $\begin{array}{l}\text { Hospital admission in } 2 \mathrm{yr} \\
\text { preceding index test }\end{array}$ & $22600(5.2)$ & $17701(5.1)$ & $4899(5.3)$ \\
\hline Urban residence & $378822(86.5)$ & $299480(86.5)$ & $79342(86.5)$ \\
\hline
\end{tabular}


Table 3: Provider and patient characteristics associated with repeat antinuclear antibody testing within 12 months of previous test and repeat testing after a positive test result

\begin{tabular}{|c|c|c|c|c|}
\hline \multirow[b]{2}{*}{ Characteristic } & \multicolumn{2}{|c|}{ Any physician, adjusted OR $(95 \% \mathrm{Cl})^{*}$} & \multicolumn{2}{|c|}{ Same physician, adjusted OR $(95 \% \mathrm{Cl})^{*}$} \\
\hline & $\begin{array}{l}\text { Repeat testing within } \\
12 \text { mo of previous } \\
\text { test }\end{array}$ & $\begin{array}{l}\text { Repeat testing after } \\
\text { prior positive test } \\
\text { result }\end{array}$ & $\begin{array}{c}\text { Repeat testing within } \\
12 \text { mo of previous } \\
\text { test }\end{array}$ & $\begin{array}{l}\text { Repeat testing after } \\
\text { prior positive test } \\
\text { result }\end{array}$ \\
\hline \multicolumn{5}{|l|}{ Physicians } \\
\hline $\begin{array}{l}\text { Family physicians (reference }= \\
\text { rheumatologists) }\end{array}$ & $0.26(0.22-0.31)$ & $0.23(0.20-0.28)$ & $0.80(0.64-1.00)$ & $0.55(0.44-0.69)$ \\
\hline $\begin{array}{l}\text { Internists (reference = } \\
\text { rheumatologists) }\end{array}$ & $0.59(0.44-0.79)$ & $0.58(0.44-0.76)$ & $0.63(0.47-0.85)$ & $0.66(0.50-0.87)$ \\
\hline $\begin{array}{l}\text { All other practitioners (reference }= \\
\text { rheumatologists) }\end{array}$ & $0.39(0.32-0.48)$ & $0.33(0.26-0.42)$ & $0.63(0.47-0.84)$ & $0.56(0.42-0.73)$ \\
\hline $\begin{array}{l}\text { Physician age } \leq 50 \text { year } \text { (reference }= \\
>50 \mathrm{yr} \text { ) }\end{array}$ & $0.98(0.88-1.10)$ & $0.90(0.79-1.03)$ & $1.29(1.15-1.46)$ & $1.12(0.97-1.29)$ \\
\hline Female $($ reference $=$ male $)$ & $0.93(0.84-1.03)$ & $1.05(0.93-1.19)$ & $0.95(0.85-1.07)$ & $1.10(0.96-1.27)$ \\
\hline $\begin{array}{l}\text { Academic centre (reference }= \\
\text { community practice) }\end{array}$ & $1.53(0.87-2.69)$ & $1.32(0.83-2.12)$ & $1.53(0.94-2.48)$ & $1.33(0.89-1.98)$ \\
\hline $\begin{array}{l}\text { International medical school graduate } \\
\text { (reference = Canadian medical school } \\
\text { graduate) }\end{array}$ & $0.96(0.87-1.07)$ & $0.91(0.80-1.05)$ & $0.81(0.70-0.93)$ & $0.75(0.63-0.88)$ \\
\hline \multicolumn{5}{|l|}{ Patients } \\
\hline Age & $1.01(1.00-1.01)$ & $1.00(1.00-1.01)$ & $1.01(1.01-1.01)$ & $1.01(1.00-1.01)$ \\
\hline Female $($ reference $=$ male $)$ & $1.29(1.25-1.34)$ & $1.82(1.73-1.91)$ & $1.29(1.23-1.36)$ & $1.83(1.72-1.94)$ \\
\hline \multicolumn{5}{|l|}{ Income quintile† (reference = 1 lowest) } \\
\hline 2 & $1.03(0.99-1.07)$ & $1.06(1.00-1.12)$ & $1.05(1.00-1.10)$ & $1.06(0.99-1.15)$ \\
\hline 3 & $1.05(1.01-1.10)$ & $1.12(1.06-1.19)$ & $1.04(0.99-1.09)$ & $1.10(1.02-1.19)$ \\
\hline 4 & $1.07(1.03-1.12)$ & $1.19(1.12-1.26)$ & $1.04(0.99-1.10)$ & $1.16(1.07-1.25)$ \\
\hline 5 (highest) & $1.08(1.03-1.12)$ & $1.17(1.10-1.25)$ & $1.02(0.97-1.07)$ & $1.10(1.01-1.19)$ \\
\hline Urban residence (reference $=$ rural) & $0.93(0.86-0.99)$ & $0.96(0.89-1.05)$ & $1.01(0.93-1.09)$ & $1.03(0.94-1.13)$ \\
\hline Connective tissue disease & $2.20(2.01-2.41)$ & $4.18(3.70-4.73)$ & $3.08(2.70-3.51)$ & $5.37(4.69-6.14)$ \\
\hline Hospital admission in previous 6 mo & $0.95(0.89-1.00)$ & $0.92(0.83-1.02)$ & $0.92(0.84-1.01)$ & $0.94(0.80-1.10)$ \\
\hline \multicolumn{5}{|l|}{$\begin{array}{l}\text { Charlson Comorbidity Index score } \\
\text { (reference }=0 \text { ) }\end{array}$} \\
\hline 1 & $1.17(1.10-1.25)$ & $1.14(1.03-1.26)$ & $1.20(1.10-1.30)$ & $1.18(1.04-1.34)$ \\
\hline$\geq 2$ & $1.11(1.02-1.21)$ & $0.97(0.86-1.09)$ & $1.16(1.05-1.29)$ & $0.98(0.85-1.13)$ \\
\hline \multicolumn{5}{|l|}{ Year of index test (reference $=2010$ ) } \\
\hline 2008 & $0.24(0.21-0.28)$ & $0.19(0.16-0.24)$ & $0.27(0.22-0.32)$ & $0.23(0.17-0.30)$ \\
\hline 2009 & $0.75(0.71-0.80)$ & $0.74(0.67-0.81)$ & $0.76(0.70-0.83)$ & $0.74(0.65-0.83)$ \\
\hline 2011 & $1.09(1.03-1.16)$ & $1.16(1.07-1.25)$ & $1.06(0.99-1.13)$ & $1.08(0.97-1.20)$ \\
\hline 2012 & $1.17(1.10-1.26)$ & $1.31(1.19-1.44)$ & $1.12(1.04-1.21)$ & $1.20(1.07-1.34)$ \\
\hline 2013 & $1.30(1.22-1.39)$ & $1.32(1.21-1.46)$ & $1.26(1.16-1.37)$ & $1.33(1.17-1.50)$ \\
\hline 2014 & $1.45(1.36-1.56)$ & $1.44(1.32-1.58)$ & $1.39(1.27-1.52)$ & $1.36(1.21-1.51)$ \\
\hline 2015 & $1.70(1.56-1.86)$ & $1.68(1.50-1.88)$ & $1.79(1.59-2.01)$ & $1.80(1.54-2.09)$ \\
\hline \multicolumn{5}{|c|}{$\begin{array}{l}\text { Note: } \mathrm{Cl}=\text { confidence interval, } \mathrm{OR}=\text { odds ratio. } \\
\text { *Adjusted for patient covariates (age, sex, income quintile, urban residence, year of testing, prior hospital admissions, Charlson Comorbidity Index score, testing or } \\
\text { diagnosis for connective tissue disease) and physician covariates (specialty, age, sex, international medical graduate, academic setting). } \\
\text { †Based on patients' postal code and census neighbourhood income quintile. } \\
\text { †With a look-back period of } 2 \text { years before the index test. }\end{array}$} \\
\hline
\end{tabular}




\section{Interpretation}

In our sample, over a quarter of all ANA tests were repeat tests, with a substantial number of potentially redundant tests. Half of repeat tests were performed within 12 months of the previous test. Among the tests repeated within 12 months, the result of the previous test was positive for $31 \%$. Family physicians ordered the most ANA tests; however, rheumatologists were more likely to order repeat tests and repeat testing after a positive result than other practitioners. The most significant correlate of potentially redundant testing involved testing in patients with suspected or confirmed connective tissue disease. Moreover, the volume of ANA testing far exceeded the number of expected new cases of connective tissue disease, ${ }^{24-26}$ which raises concerns of potential overuse of ANA testing.

Our findings are consistent with previous studies showing that ANA testing is pervasive in rheumatology practice. ${ }^{22,23,27,28,30-32}$ We observed similar frequencies of ANA positivity in our sample and similarly identified that family physicians order the majority of ANA tests. ${ }^{27,31}$ Although there are far more family physicians than other specialists, it has been suggested that family physicians have limited expertise in interpreting ANA tests and may ignore results. ${ }^{27}$

Our findings show that rheumatologists were most likely to order repeat testing, which is consistent with a recent Canadian study. ${ }^{27}$ However, the proportion of potentially redundant repeat tests in our sample is higher than in previous studies. ${ }^{27,31,33}$ Potential explanations for this may be our ability to capture most tests performed across settings in Ontario and the publicly funded health care system in which this study was performed (i.e., patients not required to pay out of pocket for testing).

There are several potential reasons why rheumatologists order repeat tests, including issues surrounding access to and perceived accuracy of previous results, testing for research participants and the introduction of biologic therapy for immune-mediated diseases, which may drive ANA testing for drug-induced systemic lupus. Yet a recent Canadian survey showed that many rheumatologists feel they are ordering ANA tests correctly and that Choosing Wisely recommendations do not apply to them since only family physicians order ANA tests inappropriately. ${ }^{34}$ In one Canadian city, rheumatologists were found to be the third-highest laboratory spenders per physician by specialty, ${ }^{35}$ which raises concerns over the volume of laboratory testing in their patients.

Unnecessary test repetition is readily modifiable both through increasing education and awareness of overuse, and by enhancing access to outside health records and sharing results. ${ }^{5}$ Targeted strategies are effective in improving the appropriateness of testing. ${ }^{33}$ Multiple linked interventions coupled with computerized order set modifications can effect lasting change in ordering behaviours. ${ }^{36}$

It is difficult to extrapolate costs associated with repeat testing. In Ontario, an ANA test costs $\$ 6.85,{ }^{37}$ which equates to about $\$ 1.1$ million for the 164913 repeat tests performed during the study period. In addition to the direct costs related to the test itself, there are direct labour costs (e.g., phlebotomists), indirect labour costs (e.g., administrative), direct material costs (e.g., collection needles, tubes) and indirect material costs (e.g., facilities, analyzer). Potential downstream costs, such as unnecessary specialist consultations, may also be incurred.

\section{Limitations}

We did not have clinical data to inform reasons for repeat testing. As it is difficult to standardize ANA tests between laboratories, we used each laboratory's reported interpretation (positive or negative) and thus were unable to assess ANA titres, which could potentially influence physicians' ordering of repeat testing. We did not assess ANA subserology.

We were also unable to assess testing that was ordered but not carried out by the patient. We did not study underscreening in targeted populations, which is another form of poor quality of care. A limitation of the laboratory data used in this study is the incompleteness of data coverage in the earliest years of our study period. Thus, we may have underestimated the frequency of repeat testing if patients had tests conducted at laboratories not captured in our source databases. Moreover, our primary outcome was a repeat test within 12 months of the previous test, and the first ANA test appearing in our data source may not have been a patient's first test.

\section{Conclusion}

We identified a substantial number of potentially redundant ANA tests. Our findings have implications for qualityimprovement initiatives related to the appropriateness of ANA testing. These may include developing strategies to support both primary care physicians (to ensure that appropriate patients undergo testing) and rheumatologists (to limit repeat testing). Health policy changes could eliminate duplicate orders for tests within specified time frames. In Canada, certain rheumatologic tests can be ordered only by specialists, to reduce unnecessary testing. Further research is also required to understand why physicians order ANA tests and repeat tests, and to explore ANA subserology testing patterns.

\section{References}

1. McGregor MJ, Martin D. Testing 1, 2, 3: Is overtesting undermining patient and system health? Can Fam Physician 2012;58:1191-3, e615-7.

2. McGrail KM, Evans RG, Barer ML, et al. Diagnosing senescence: contributions to physician expenditure increases in British Columbia, 1996/97 to 2005/06. Healthc Policy 2011;7:41-54.

3. Sivananthan SN, Peterson S, Lavergne R, et al. Designation, diligence and drift: understanding laboratory expenditure increases in British Columbia, 1996/97 to 2005/06. BMC Health Serv Res 2012;12:472.

4. Zhi M, Ding EL, Theisen-Toupal J, et al. The landscape of inappropriate laboratory testing: a 15-year meta-analysis. PLoS One 2013;8:e78962.

5. Lyu H, Xu T, Brotman D, et al. Overtreatment in the United States. PLoS One 2017;12:e0181970.

6. Bates DW, Goldman L, Lee TH. Contaminant blood cultures and resource utilization. The true consequences of false-positive results. FAMA 1991;265: 365-9.

7. Fritzler MJ. Choosing Wisely: review and commentary on anti-nuclear antibody (ANA) testing. Autoimmun Rev 2016;15:272-80.

8. Chow SL, Carter Thorne J, Bell MJ, et al. Choosing Wisely: the Canadian Rheumatology Association's list of 5 items physicians and patients should question. 7 Rheumatol 2015;42:682-9.

9. Yazdany J, Schmajuk G, Robbins M, et al. Choosing Wisely: the American College of Rheumatology's Top 5 list of things physicians and patients should question. Arthritis Care Res (Hoboken) 2013;65:329-39. 
10. Thomas C, Robinson JA. The antinuclear antibody test. When is a positive result clinically relevant? Postgrad Med 1993;94:55-8, 63, 66.

11. Shojania K. Rheumatology: 2. What laboratory tests are needed? CMA7 2000; 162:1157-63.

12. Castro C, Gourley M. Diagnostic testing and interpretation of tests for autoimmunity. 7 Allergy Clin Immunol 2010;125(Suppl 2):S238-47.

13. Wandstrat AE, Carr-Johnson F, Branch V, et al. Autoantibody profiling to identify individuals at risk for systemic lupus erythematosus. 7 Autoimmun 2006;27:153-60.

14. Solomon DH, Kavanaugh AJ, Schur PH, et al. Evidence-based guidelines for the use of immunologic tests: antinuclear antibody testing. Arthritis Rheum 2002;47:434-44.

15. Raissi TC, Hewson C, Pope JE. Repeat testing of antibodies and complements in systemic lupus erythematosus: When is it enough? 7 Rheumatol 2018;45: 827-34.

16. Agmon-Levin N, Damoiseaux J, Kallenberg C, et al. International recommendations for the assessment of autoantibodies to cellular antigens referred to as anti-nuclear antibodies. Ann Rheum Dis 2014;73:17-23.

17. Five things physicians and patients should question [Choosing Wisely]. Philadelphia: American College of Rheumatology; 2013. Available: www. choosingwisely.org/societies/american-college-of-rheumatology-pediatric -rheumatology/ (accessed 2019 July 9).

18. Kavanaugh A, Tomar R, Reveille J, et al. Guidelines for clinical use of the antinuclear antibody test and tests for specific autoantibodies to nuclear antigens. American College of Pathologists. Arch Pathol Lab Med 2000;124:71-81.

19. Fitch-Rogalsky C, Steber W, Mahler M, et al. Clinical and serological features of patients referred through a rheumatology triage system because of positive antinuclear antibodies. PLoS One 2014;9:e93812.

20. Narain S, Richards HB, Satoh M, et al. Diagnostic accuracy for lupus and other systemic autoimmune diseases in the community setting. Arch Intern Med 2004;164:2435-41.

21. Rolfe A, Burton C. Reassurance after diagnostic testing with a low pretest probability of serious disease: systematic review and meta-analysis. $7 A M A$ Intern Med 2013;173:407-16.

22. Mohammed AS, Boddu P, Mael D, et al. Inappropriate use of commercial antinuclear antibody testing in a community-based US hospital: a retrospective study. 7 Community Hosp Intern Med Perspect 2016;6:32031.

23. Mohammadi S, Shaik I, Chevli P, et al. Improper use of antinuclear antibody (ANA) test can result in misdiagnosis, increased patient anxiety, and wasted health care resources [abstract]. Proceedings from the 2014 ACR/ARHP Annual Meeting; 2014 Nov. 14-19; Boston. Arthritis Rheumatol 2014:66.

24. Ungprasert P, Sagar V, Crowson CS, et al. Incidence of systemic lupus erythematosus in a population-based cohort using revised 1997 American College of Rheumatology and the 2012 Systemic Lupus International Collaborating Clinics classification criteria. Lupus 2017;26:240-7.

25. Fatoye F, Gebrye T, Svenson LW. Real-world incidence and prevalence of systemic lupus erythematosus in Alberta, Canada. Rheumatol Int 2018;38:1721-6.

26. Mayes MD. Scleroderma epidemiology. Rheum Dis Clin North Am 2003;29: 239-54.

27. Man A, Shojania K, Phoon C, et al. An evaluation of autoimmune antibody testing patterns in a Canadian health region and an evaluation of a laboratory algorithm aimed at reducing unnecessary testing. Clin Rheumatol 2013;32:601-8.

28. Abeles AM, Abeles $M$. The clinical utility of a positive antinuclear antibody test result. Am 7 Med 2013;126:342-8.

29. Kralj B. Measuring 'rurality' for purposes of health-care planning: an empirical measure for Ontario. Ont Med Rev 2000;67:33-52.

30. Lake S, Gakhal N, Steiman A, et al. The frequency and cost of repeat ANA testing at two University of Toronto-affiliated teaching hospitals. 7 Rheumatol 2018;45:1036-7.
31. Davis LA, Goldstein B, Tran V, et al. Applying Choosing Wisely: antinuclear antibody (ANA) and sub-serology testing in a safety net hospital system. Open Rheumatol 7 2015;9:82-7.

32. Bulbin D, Meadows A, Denio A, et al. Abstracts of the 2013 Annual Meeting of the American College of Rheumatology. October 25-30, 2013. San Diego, California, USA. Do rheumatologists (and other specialists) practice what we preach? A study of serology ordering patterns with attention to subserologies when the antinuclear antibody by enzyme linked immunosorbent assay is negative; and the clinical significance of these positive subserology results [abstract]. Arthritis Rheum 2013;65:S1-1331.

33. Lesuis N, Hulscher ME, Piek E, et al. Choosing Wisely in daily practice: an intervention study on antinuclear antibody testing by rheumatologists. Arthritis Care Res (Hoboken) 2016;68:562-9.

34. Zeiadin N, Averns H. CRA survey results: Choosing Wisely. Can Rheumatol Assoc 7 2018;28:26-8.

35. Naugler C, Thomas R, Turin TC, et al. Yearly clinical laboratory test expenditures for different medical specialties in a major Canadian city. Am 7 Clin Pathol 2015;144:97-102.

36. Sadowski BW, Lane AB, Wood SM, et al. High-value, cost-conscious care: iterative systems-based interventions to reduce unnecessary laboratory testing. Am 7 Med 2017;130:1112.e1-1112.e7.

37. Ontario Health Insurance Plan: OHIP Schedule of Benefits and Fees. Toronto: Ministry of Health and Long-Term Care; 2019. Available: http:// health.gov.on.ca/en/pro/programs/ohip/sob/ (accessed 2019 Aug. 14).

Affiliations: Holland Bone \& Joint Program (Lake, Widdifield), Sunnybrook Research Institute; ICES (Yao, Hawker, Widdifield); Women's College Hospital (Gakhal, Hawker); Sinai Health System (Steiman) and Institute of Health Policy, Management \& Evaluation (Hawker, Widdifield), University of Toronto, Toronto, Ont.

Contributors: Jessica Widdifield, Shirley Lake, Amanda Steiman and Natasha Gakhal conceived the study. Gillian Hawker, Jessica Widdifield and Zhan Yao acquired the data. Zhan Yao analyzed the data. Shirley Lake and Jessica Widdifield drafted the manuscript. All of the authors contributed to the study design and to data interpretation, revised the manuscript critically for important intellectual contact, approved the final version to be published and agreed to be accountable for all aspects of the work.

Funding: This study was supported by the University of Toronto Pfizer Chair in Rheumatology Research Award and by ICES, which is funded by an annual grant from the Ontario Ministry of Health and Long-Term Care.

Disclaimer: This study was supported by ICES, which is funded by an annual grant from the Ontario Ministry of Health and Long-Term Care. Parts of this material are based on data and information compiled and provided by the Canadian Institute for Health Information. The analyses, conclusions, opinions and statements expressed herein are solely those of the authors and do not reflect those of the funding or data sources; no endorsement is intended or should be inferred.

Supplemental information: For reviewer comments and the original submission of this manuscript, please see www.cmajopen.ca/content/8/1/ E184/suppl/DC1. 